\title{
Perfil hematológico de equinos submetidos à prova de Team Penning ${ }^{1}$
}

\author{
Renata Lima de Miranda ${ }^{2 *}$, Antonio Vicente Mundim³ ${ }^{3}$ Ana Carolina Silveira \\ Saquy $^{3}$, Álisson Souza Costa ${ }^{3}$, Ednaldo Carvalho Guimarães ${ }^{3}$, Felipe César \\ Gonçalves $^{3}$ e Frederico Ozanam Carneiro e Silva ${ }^{3}$
}

\begin{abstract}
Miranda R.L., Mundim A.V., Saquy A.C.S, Costa A.S., Guimarães E.C., Gonçalves F.C. \& Silva F.O.C. 2011. [Hematologic profile of horses subjected to Team Penning.] Perfil hematológico de equinos submetidos à prova de Team Penning. Pesquisa Veterinária Brasileira 31(1):81-86. Laboratório Clínico e de Anatomia Animal, Faculdade de Medicina Veterinária, Universidade Federal de Uberlândia, Av. Pará 1720, Bloco 2T, Campus Umuarama, Uberlândia, MG 38400-902, Brazil. E-mail: renatavetufu@ yahoo.com.br

Variations in hematologic parameters are used to assess the degree of training or clinical state of the animal. The hematologic evaluation of horses at rest has been an object of study in order to establish a correlation with training or athletic capacity. The purpose of this study was to evaluate the hematologic profile of horses subjected to Team Penning competitions, correlating sex and frequency of physical activity. Two milliliters of blood were drawn through a puncture made in the external jugular vein from 29 horses, 18 males and 11 females, at rest (Moment I) and after exercising (Moment II). The blood samples were processed in an $A B C$ VET automated veterinary hematology analyzer (Horiba ABX Diagnostics). The animals were divided into Group A, B, C and D according to the number of times they participated in the competition. The values of globular volume, hemoglobin, erythrocytes, rod-shaped segmented neutrophils and monocytes increased after the physical exercise, unlike the number of lymphocytes and eosinophils, which decreased. A comparison of the before/after exercise correlations showed no significant differences $(p<0.05)$ between males and females. In addition, it was found that the value of the MI/MII ratio for the globular volume, hemoglobin and number of erythrocytes varied according to the frequency of the exercise. It was concluded that the Team Penning competition produces hematologic alterations in horses, which are affected by the frequency of exercising, regardless of sex.
\end{abstract}

INDEX TERMS: Equus caballus, exercise, hematology.

RESUMO.- As variações nos parâmetros hematológicos são utilizadas com intuito de avaliar o grau de treinamento ou estado clínico do animal. A avaliação hematológica de eqüinos em repouso tem sido objeto de estudo, a fim de estabelecer uma relação com treinamento ou capacidade atlética. Objetivou-se avaliar o perfil hematológico de eqüinos submetidos à prova de Team Penning, correlacio-

\footnotetext{
${ }^{1}$ Recebido em 9 de setembro de 2009.

Aceito para publicação em 25 de novembro de 2010

2 Doutoranda em Imunologia e Parasitologia Aplicadas da Universidade Federal de Uberlândia (UFU), Uberlândia, MG, Brasil. *Autor para correspondência: renatavetufu@yahoo.com.br

${ }^{3}$ Laboratório Clínico e de Anatomia Animal, Faculdade de Medicina Veterinária, UFU, Av. Pará 1720, Bloco 2T, Campus Umuarama, Uberlândia, MG 38400-902.
}

nando o sexo e freqüência da atividade física. Mediante punção da veia jugular externa coletaram-se dois $\mathrm{mL}$ de sangue de 29 eqüinos, 18 machos e 11 fêmeas, em repouso (Momento I) e após o exercício (Momento II). As amostras de sangue foram processadas em analisador hematológico automático veterinário ( $A B C$ VET - Horiba $A B X$ Diagnostics). Os animais foram divididos em Grupos A, B, $\mathrm{C}$ e $\mathrm{D}$, de acordo com o número de participações na prova. Observou-se que os valores de volume globular, hemoglobina, hemácias, leucócitos, neutrófilos em bastonetes e segmentados, e monócitos aumentaram após o exercício físico, ao contrário do número de linfócitos e eosinófilos, que reduziram. Não existiram diferenças significativas $(p<0,05)$ entre machos e fêmeas ao confrontar as relações antes/depois. Além disso, evidenciou-se que o valor da 
relação MI/MII para volume globular, hemoglobina e número de hemácias variou de acordo com a freqüência do exercício. Conclui-se que a prova de Team Peninng ocasiona alterações hematológicas em eqüinos, com interferência da freqüência do exercício, independente do sexo.

TERMOS DE INDEXAÇÃO: Equus caballus, exercício, hematologia.

\section{INTRODUÇÃO}

As variações no perfil hematológico são utilizadas para avaliação de treinamento ou estado clínico. A avaliação hematológica de eqüinos em repouso tem sido objeto de estudo visando estabelecer uma relação com treinamento ou capacidade atlética (Rose et al.1983).

De acordo com Hanzawa et al. (1999), o exercício aeróbico afeta os eritrócitos sanguíneos mediante alterações na composição lipídica e na estrutura protéica da membrana celular, que acabam reduzindo a fragilidade osmótica dos eritrócitos. Enquanto que o exercício anaeróbico torna os eritrócitos suscetíveis às variações osmóticas, ou seja, aumenta a fragilidade osmótica em eqüinos atletas.

Resposta à excitação é uma alteração imediata associada à liberação de epinefrina. Isso resulta em eventos cardiovasculares que, por sua vez aumentam o fluxo sanguíneo da microcirculação, principalmente nos músculos. O exercício extenuante antes da crise hemorrágica pode ter o mesmo efeito. Isso resulta na migração de leucócitos do compartimento marginal para o circulante. No leucograma, nota-se, aproximadamente, o dobro da quantidade de leucócitos, devido ao aumento de neutrófilos e/ou linfócitos. Não ocorre desvio a esquerda, porque a neutrofilia é decorrente do aumento da população de células maduras na microcirculação, que alcançam o compartimento circulante (Thrall et al. 2007).

Já o estresse fisiológico, em resposta, principalmente, à distúrbios metabólicos (desidratação) e dor, ocorre devido à liberação de hormônio adrenocorticotrópico pela glândula hipófise e conseqüente liberação de cortisol pela glândula adrenal. A principal alteração é linfopenia. Os esteróides podem induzir apoptose de linfócitos e alterar seu padrão de recirculação. A segunda alteração mais consistente é a duplicação da população de neutrófilos circulantes. Não há desvio a esquerda, a menos que haja uma doença inflamatória simultânea. Eosinopenia é a terceira alteração mais comum e ocorrência de monocitose é variável (Thrall et al. 2007).

A resposta hematológica frente ao exercício físico é considerada conseqüência dos níveis plasmáticos aumentados de cortisol, decorrente do estresse e está correlacionada com a concentração de adrenalina, velocidade e freqüência cardíaca do animal. O exercício provoca aumento transitório da concentração plasmática de catecolaminas, ACTH e cortisol em reposta ao eixo hipotálamohipófise-suprarenal. As catecolaminas promovem mobilização de eritrócitos e linfócitos provenientes do baço.
Enquanto o ACTH e cortisol estimulam a produção de neutrófilos e migração de granulócitos para os tecidos. A contagem de leucócitos pode aumentar entre 10 e 30\% dependendo da intensidade e duração do exercício (Santos 2006). Os parâmetros hematológicos podem ser influenciados pela raça, idade, sexo e alimentação, além do exercício físico (Piccione et al. 2001).

Análises laboratoriais tornaram-se fundamentais na avaliação do eqüino em competição, transformando-se em ferramentas decisivas para o acompanhamento do animal atleta (Balarin et al. 2005). Portanto, as alterações hematológicas que ocorrem em conseqüência do exercício e a sua importância na avaliação da intensidade do esforço físico, nortearam a realização desta pesquisa. Cujo objetivo foi avaliar o perfil hematológico, correlacionando o sexo e freqüência da atividade física, em eqüinos submetidos à prova de Team Penning.

\section{MATERIAL E MÉTODOS}

O experimento foi realizado no Parque de Exposições (CAMARU) na cidade de Uberlândia, MG, durante a etapa final da modalidade Team Penning. Participaram 29 equinos, mestiços Quarto de Milha, de 4-9 anos de idade, sendo 18 machos e 11 fêmeas.

Realizaram-se duas coletas de sangue em cada animal. A primeira pela manhã antes da competição com o animal em repouso (Momento I) e a segunda 20 minutos após a última entrada do animal na pista para competição (Momento II). Em cada momento foram coletados dois $\mathrm{mL}$ de sangue em tubo a vácuo com ácido etilenodiaminotetraacético (EDTA) (BD Vacutainer®), por venipunção da jugular externa, os quais foram utilizados no processamento do hemograma.

Após coletadas, as amostras foram imediatamente encaminhadas ao Laboratório Clínico Veterinário do Hospital Veterinário da UFU, onde foram processadas em analisador hematológico automático veterinário ( $A B C$ VET, Horiba $A B X$ Diagnostics). Em que foi possível determinar os valores de volume globular, hemoglobina, número total de leucócitos, hemácias, plaquetas, volume globular médio (VGM), concentração da hemoglobina globular média (CHGM) e amplitude da distribuição da série vermelha (RDW). A contagem diferencial complementar de leucócitos foi feita a partir de extensões sanguíneas coradas com May-Grünwald-Giemsa (MGG), segundo Ferreira Neto et al. (1982), em que se estabeleceu a percentagem (valor relativo) de monócitos, linfócitos, eosinófilos e neutrófilos em bastonetes e segmentados, com a contagem de 100 células. Conseqüentemente, ao multiplicá-las pela contagem total de leucócitos, obtiveram-se os valores absolutos de cada tipo celular.

Os animais foram agrupados de acordo com o número de participações na prova (Quadro 1).

A análise estatística de cada variável analisada se baseou em três testes, com nível de significância de 5\%, de acordo com Ayres et al. (2005). Aplicou-se o teste paramétrico $t$ de Student de comparação de médias para amostras dependentes, ou seja, foi verificado se as variáveis analisadas apresentaram resultados estatisticamente iguais antes (Momento I) e após (Momento II) o exercício. Utilizou-se do mesmo teste, porém para amostras independentes, a fim de averiguar se a relação antes/depois dos atributos estudados apresentou resultados 
Quadro 1. Grupos de animais de acordo com o número de participações na competição Team Penning

\begin{tabular}{ccc}
\hline Grupo & № de participações & № de animais \\
\hline A & 1 a 5 & 7 \\
B & 6 a 10 & 8 \\
C & 11 a 15 & 11 \\
D & Acima de 16 & 3 \\
Total & & 29
\end{tabular}

equivalentes quanto a machos e fêmeas. A análise de variância em delineamento inteiramente ao acaso com aplicação do teste de Tukey foi utilizada com intuito de verificar a existência de diferença significativa entre as relações antes/depois dos Grupos A, B, C e D para cada atributo analisado. Trabalho submetido e aprovado pelo Comitê de Ética na utilização de Animais da Universidade Federal de Uberlândia (CEUA/UFU) sob o parecer número 019/09.

\section{RESULTADOS}

Os valores de volume globular, hemoglobina, hemácias, leucócitos, neutrófilos em bastonetes e segmentados, e monócitos aumentaram $(p<0,05)$ após o exercício físico. Entretanto o número de linfócitos e eosinófilos reduziram em conseqüência da atividade física (Quadro 2).

Confrontados os valores dos parâmetros hematológicos dos animais em repouso e após o exercício com os valores de referência citados Jain (1993), observou-se que a maioria deles permaneceu dentro dos limites citados pelo autor, exceto, os neutrófilos em bastonetes, os quais apresentaram seus valores ligeiramente acima do fisiológico.

Evidenciou-se redução do valor da relação MI/MII para volume globular, hemoglobina e número de hemácias nos equinos com mais de seis participações na prova, comparados aqueles com até cinco participações (Quadro 3). Não existiram diferenças significativas ao confrontar as relações antes/depois de machos e fêmeas (Quadro 4).
Quadro 2. Médias e desvios padrão dos parâmetros hematológicos dos animais em repouso (Momento I) e após exercício (Momento II)

\begin{tabular}{|c|c|c|c|c|}
\hline \multirow[t]{2}{*}{ Parâmetros } & \multicolumn{2}{|c|}{ Momento I $\left(n^{c}=29\right)$} & \multicolumn{2}{|c|}{ Momento II $(n=29)$} \\
\hline & Média & $\begin{array}{l}\text { Desvio } \\
\text { padrão }\end{array}$ & Média & $\begin{array}{l}\text { Desvio } \\
\text { padrão }\end{array}$ \\
\hline Volume globular (\%) & $34,86^{b}$ & 3,29 & $39,00^{a}$ & 3,64 \\
\hline Hemoglobina $(\mathrm{g} / \mathrm{dL})$ & $11,54^{b}$ & 1,04 & $12,84^{a}$ & 1,14 \\
\hline Hemácias (x 106/ $\mu \mathrm{L})$ & $7,69^{b}$ & 0,77 & $8,58^{a}$ & 0,86 \\
\hline $\operatorname{VGM}\left(\mu \mathrm{m}^{3}\right)$ & $45,41^{a}$ & 2,06 & $45,44^{a}$ & 1,90 \\
\hline CHGM (g/dL) & 33,1 & 0,56 & $32,96^{a}$ & 0,44 \\
\hline RDW (\%) & $17,83^{a}$ & 0,75 & $17,78^{a}$ & 0,61 \\
\hline Plaquetas (x $\left.10^{3} / \mu \mathrm{L}\right)$ & $143,27^{a}$ & 50,95 & $141,89^{a}$ & 46,09 \\
\hline 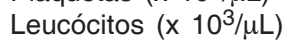 & $8,09^{b}$ & 2,01 & $11,95^{a}$ & 3,40 \\
\hline Bastonetes $(\mu \mathrm{L})$ & $132,24^{b}$ & 163,56 & $309,10^{a}$ & 358,08 \\
\hline Segmentados $(/ \mu \mathrm{L})$ & $4444,79^{b}$ & 1666,65 & $8337,17^{a}$ & 3315,45 \\
\hline Eosinófilos $(/ \mu \mathrm{L})$ & $286,31^{a}$ & 202,89 & $118,86^{b}$ & 144,06 \\
\hline Linfócitos $(/ \mu \mathrm{L})$ & $2925,13^{a}$ & 985,85 & $2574,93^{b}$ & 816,18 \\
\hline Monócitos $(/ \mu \mathrm{L})$ & $249,93^{b}$ & 172,19 & $580,68^{a}$ & 324,12 \\
\hline
\end{tabular}

Médias nas linhas seguidas por letras diferentes são estatisticamente diferentes $(p<0,05)$.

c Número de animais.

\section{DISCUSSÃO}

Neste estudo, observou-se aumento do volume globular após a prática de atividade física, fato também relatado por Snow et al. (1983a), Harris \& Snow $(1988,1992)$, McKeever et al. (1993), Andrews et al. (1995), Gómez et al. (2004), Orozco et al. (2006) e Ferraz et al. (2009). Segundo Snow et al. ( 1983a), o aumento do volume globular é resultado da contração esplênica e redução do volume plasmático por redistribuição do volume vascular, perda de fluido por meio do suor e respiração. Santos (2006) salientou que a elevação do volume globular pode se dar por perda de água do compartimento extravascular ou por troca transitória de fluidos entre o compartimento extra e intravascular. A perda de líquidos é atribuída à sudorese, principalmente em condições ambientais de calor e umi-

Quadro 3. Médias dos Momentos I e II e das relações MI/MII dos parâmetros hematológicos de equinos dos Grupos A, B, C, D

\begin{tabular}{|c|c|c|c|c|c|c|c|c|c|c|c|c|}
\hline \multirow[t]{2}{*}{ Parâmetros } & \multicolumn{3}{|c|}{ Grupo A } & \multicolumn{3}{|c|}{ Grupo B } & \multicolumn{3}{|c|}{ Grupo C } & \multicolumn{3}{|c|}{ Grupo D } \\
\hline & $\begin{array}{c}\text { Média } \\
\text { MIc }\end{array}$ & $\begin{array}{c}\text { Média } \\
\text { MIId }\end{array}$ & $\begin{array}{l}\text { Média } \\
\text { MI/MII }\end{array}$ & $\begin{array}{c}\text { Média } \\
\text { MI }\end{array}$ & $\begin{array}{l}\text { Média } \\
\text { MII }\end{array}$ & $\begin{array}{l}\text { Média } \\
\text { MI/MII }\end{array}$ & $\begin{array}{c}\text { Média } \\
\text { MI }\end{array}$ & $\begin{array}{c}\text { Média } \\
\text { MII }\end{array}$ & $\begin{array}{l}\text { Média } \\
\text { MI/MII }\end{array}$ & $\begin{array}{c}\text { Média } \\
\text { MI }\end{array}$ & $\begin{array}{c}\text { Média } \\
\text { MII }\end{array}$ & $\begin{array}{l}\text { Média } \\
\text { MI/MII }\end{array}$ \\
\hline Volume globular (\%) & 35,87 & 35,70 & $1.00^{\mathrm{a}}$ & 33,80 & 39,41 & $0.85^{b}$ & 34,84 & 39,91 & $0.87^{b}$ & 35,50 & 42,27 & $0.83^{b}$ \\
\hline Hemoglobina (g/dL) & 11,94 & 11,87 & $1.00^{\mathrm{a}}$ & 11,25 & 12,99 & $0.86^{b}$ & 11,47 & 13,08 & $0.88^{b}$ & 11,67 & 13,90 & $0.84^{b}$ \\
\hline 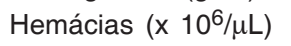 & 7,85 & 7,80 & $1.00^{\mathrm{a}}$ & 7,47 & 8,69 & $0.86^{b}$ & 7,65 & 8,75 & $0.87^{b}$ & 8,11 & 9,56 & $0.85^{a b}$ \\
\hline VGM $\left(\mu \mathrm{m}^{3}\right)$ & 45,71 & 45,71 & $1.00^{\mathrm{a}}$ & 45,50 & 45,38 & $1.00^{\mathrm{a}}$ & 45,64 & 45,64 & $1.00^{\mathrm{a}}$ & 43,67 & 44,33 & $0.98^{a}$ \\
\hline 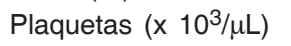 & 142,14 & 141,00 & $1.06^{a}$ & 127,50 & 143,38 & $0.91^{a}$ & 151,82 & 145,36 & $1.09^{a}$ & 156,67 & 127,33 & $1.23^{\mathrm{a}}$ \\
\hline 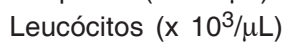 & 8,59 & 11,41 & $0.77^{a}$ & 7,13 & 10,79 & $0.68^{a}$ & 8,70 & 12,95 & $0.70^{\mathrm{a}}$ & 7,30 & 12,67 & $0.56^{a}$ \\
\hline Bastonetes $(/ \mu \mathrm{L})$ & 161,71 & 290,29 & $0.85^{a}$ & 109,63 & 180,50 & $0.45^{\mathrm{a}}$ & 147,91 & 423,27 & $0.87^{a}$ & 66,33 & 277,33 & $0.19^{a}$ \\
\hline Segmentados $(/ \mu \mathrm{L})$ & 5008,57 & 7704,86 & $0.70^{\mathrm{a}}$ & 3629,25 & 7202,13 & $0.54^{a}$ & 4874,82 & 9180,91 & $0.57^{a}$ & 3727,33 & 9745,67 & $0.37^{a}$ \\
\hline 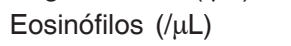 & 334,57 & 149,00 & $2.16^{a}$ & 281,25 & 78,00 & $1.57^{\mathrm{a}}$ & 238,45 & 105,82 & $1.20^{\mathrm{a}}$ & 362,67 & 205,33 & $1.09^{a}$ \\
\hline 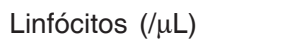 & 2688,57 & 2584,86 & $1.10^{\mathrm{a}}$ & 2865,13 & 2749,63 & $1.02^{\mathrm{a}}$ & 3106,27 & 2608,18 & $1.24^{\mathrm{a}}$ & 2973,00 & 1964,00 & $1.51^{\mathrm{a}}$ \\
\hline
\end{tabular}

Médias nas linhas seguidas por letras diferentes são estatisticamente diferentes $(p<0,05) .{ }^{c}$ Momento I (repouso), ${ }^{d}$ Momento II (após exercício). 
Grupo 4. Médias dos parâmetros hematológicos de machos e fêmeas dos Momentos I e II, com desvios padrão, e das relações MI/MII

\begin{tabular}{|c|c|c|c|c|c|c|}
\hline \multirow[t]{2}{*}{ Parâmetros } & \multicolumn{3}{|c|}{ Macho $(n=18)^{\star}$} & \multicolumn{3}{|c|}{ Fêmea $(n=11)$} \\
\hline & $\begin{array}{l}\text { Média } \\
\mathrm{MI}^{\star *}\end{array}$ & $\begin{array}{l}\text { Média } \\
\text { MII*** }\end{array}$ & $\begin{array}{l}\text { Média } \\
\text { MI/MII }\end{array}$ & $\begin{array}{c}\text { Média } \\
\text { MI }\end{array}$ & $\begin{array}{c}\text { Média } \\
\text { MII }\end{array}$ & $\begin{array}{l}\text { Média } \\
\text { MI/MI }\end{array}$ \\
\hline Volume globular (\%) & $34,26 \pm 3,41$ & $38,08 \pm 3,40$ & $0,90^{\mathrm{a}}$ & $35,85 \pm 2,97$ & $40,49 \pm 3,68$ & $0,89^{a}$ \\
\hline Hemoglobina (g/dL) & $11,38 \pm 1,07$ & $12,56 \pm 1,07$ & $0,90^{\mathrm{a}}$ & $11,80 \pm 0,99$ & $13,31 \pm 1,14$ & $0,89^{a}$ \\
\hline Hemácias (x $\left.10^{6} / \mathrm{iL}\right)$ & $7,54 \pm 0,85$ & $8,40 \pm 0,85$ & $0,90^{\mathrm{a}}$ & $7,93 \pm 0,57$ & $8,89 \pm 0,83$ & $0,90^{\mathrm{a}}$ \\
\hline VGM $\left(\mathrm{im}^{3}\right)$ & $45,55 \pm 2,03$ & $45,33 \pm 1,74$ & $1,00^{\mathrm{a}}$ & $45,18 \pm 2,18$ & $45,63 \pm 2,20$ & $0,99^{a}$ \\
\hline CHGM $(\mathrm{g} / \mathrm{dL})$ & $33,21 \pm 0,64$ & $33,00 \pm 0,44$ & $1,00^{\mathrm{a}}$ & $32,92 \pm 0,36$ & $32,89 \pm 0,45$ & $1,00^{\mathrm{a}}$ \\
\hline RDW (\%) & $17,84 \pm 0,88$ & $17,85 \pm 0,67$ & $0,99^{a}$ & $17,81 \pm 0,50$ & $17,67 \pm 0,50$ & $1,00^{\mathrm{a}}$ \\
\hline Plaquetas (x $\left.10^{3} / \mathrm{iL}\right)$ & $141,33 \pm 41,00$ & $147,66 \pm 35,14$ & $0,98^{a}$ & $146,45 \pm 66,28$ & $132,45 \pm 60,75$ & $1,15^{\mathrm{a}}$ \\
\hline Leucócitos (x $\left.10^{3} / \mathrm{iL}\right)$ & $7,82 \pm 2,05$ & $11,21 \pm 2,71$ & $0,71^{a}$ & $8,53 \pm 1,93$ & $13,17 \pm 4,15$ & $0,68^{a}$ \\
\hline Bastonetes (/iL) & $163,44 \pm 198,28$ & $268,33 \pm 272,54$ & $0,95^{a}$ & $81,18 \pm 58,69$ & $375,81 \pm 474,20$ & $0,26^{a}$ \\
\hline Segmentados (/iL) & $4313,50 \pm 1663,20$ & $7690,11 \pm 2508,18$ & $0,59^{a}$ & $4659,63 \pm 1729,94$ & $9396,00 \pm 4254,02$ & $0,54^{a}$ \\
\hline Eosinófilos (/iL) & $346,16 \pm 223,27$ & $111,77 \pm 157,00$ & $2,03^{a}$ & $188,36 \pm 116,28$ & $130,45 \pm 126,38$ & $1,16^{a}$ \\
\hline Linfócitos (/ì) & $2708,72 \pm 888,76$ & $2529,94 \pm 649,80$ & $1,10^{\mathrm{a}}$ & $3279,27 \pm 1075,28$ & $2648,54 \pm 1066,67$ & $1,31^{\mathrm{a}}$ \\
\hline Monócitos (/IL & $256,22 \pm 187,04$ & $584,94 \pm 341,80$ & $0,52^{\mathrm{a}}$ & $239,63 \pm 152,85$ & $573,72 \pm 308,95$ & $0,54^{\mathrm{a}}$ \\
\hline
\end{tabular}

Médias nas linhas seguidas por letras diferentes são estatisticamente diferentes $(p<0,05)$. * Número de animais,

** Momento I (repouso), ${ }^{* * *}$ Momento II (após exercício).

dade alta. A contração esplênica também é responsável pelo aumento, sendo que o volume globular pode elevarse em $40 \%$ devido à combinação deste fator com a redistribuição do volume de fluido circulante, mediante aumento da pressão sanguínea arterial. $O$ aumento do volume globular previne a queda da concentração de oxigênio sanguíneo durante o exercício intenso.

Aumento da concentração de hemoglobina após o exercício foi relatado por Aguilera-Tejero et al. (2000), Gómez et al. (2004), Orozco (2007) e Ferraz et al. (2009). No presente estudo, isto também ocorreu, possivelmente, com o intuito de aumentar a capacidade de oxigenação do sangue como resposta fisiológica ao exercício. Gómez et al. (2004) justificaram que este aumento responde aos mesmos fatores que levam ao aumento do volume globular, ou seja, em que o estímulo simpático do exercício mediante mecanismo adrenérgico produz contração na musculatura esplênica, lançando na circulação maior número de células vermelhas e, conseqüentemente, hemoglobina. Podese verificar maior elevação na concentração de hemoglobina no Grupo D, quando comparado aos demais grupos. De acordo com Rose et al. (1983) e Voss et al. (2002), as concentrações de hemoglobina são influenciadas tanto pela intensidade do exercício quanto pela excitação individual ocasionada pelo ambiente da prova.

Boucher et al. (1981) relataram redução do VGM, porém aumento da CHGM após prova de enduro. Já Smith et al. (1989), depois de exercício de alta velocidade, e PellegriniMasini et al. (2000), após intensa atividade física, observaram elevação do VGM e redução da CHGM. Neste estudo, o exercício não interferiu nos valores de VGM e CHGM, os quais permaneceram constantes nos Grupos A, B, C e D. Segundo Muñoz et al. (2008), a diferença entre pesquisadores pode ser explicada por exercícios de diferentes intensidades, tempo de coleta das amostras sanguíneas, alimentação, procedimentos analíticos, magnitude e direção de água e íons induzidos pelo exercício.

Balarin et al. (2006) estudando equinos submetidos a exercícios de diferentes intensidades observaram aumento significante dos valores de RDW, revelando ter ocorrido alteração nos tamanho das hemácias. Smith et al. (1989) e MaClay et al. (1992) também relataram este aumento após exercício. Estes resultados sugerem, segundo Balarin et al. (2006) e Smith et al. (1989), que o aumento do tamanho dos eritrócitos após exercícios de alta intensidade seja atribuído à eritrócitos de maior tamanho liberado pelo baço. Porém, neste estudo, observou-se que o valor de RDW permaneceu inalterado depois da atividade física exercida pelos animais e sem diferenças entre os Grupos A, B, C e D. Provavelmente, a liberação de células pelo baço foi mínima e insuficiente para provocar grande heterogeneidade entre as hemácias.

Leucocitose com neutrofilia foi detectada em eqüinos após exercício por Robson et al. (2003). Para Krumrych (2006), a atividade física é considerada um fator estressante que induz leucocitose. Numerosos estudos demonstram aumento transitório dos leucócitos após o exercício (Rose 1982, Snow et al. 1983b, Iversen et al. 1994, Orozco et al. 2006, Orozco 2007, Ferraz et al. 2009). Acredita-se que este aumento pode ser chamado de pseudoleucocitose, porque esta reação não está relacionada com a produção de novas células (resultante do aumento da secreção de adrenalina) e se deve, principalmente, pelo aumento de linfócitos em conseqüência da sua introdução em sangue periférico pelo baço e em menor escala pela medula óssea e gânglios linfáticos (Iversen et al. 1994, Horohov et al. 1996).

Entretanto, observações realizadas em eqüinos submetidos a exercício mostraram que a leucocitose é caracterizada por duas fases (Rose 1982, Snow et al. 1983b, Iversen et al. 1994, Korhonen et al. 2000). Depois do aumento transitório do número de linfócitos existe outro aumento de leucócito relacionado com uma pequena linfopenia e uma forte neutrofilia, resultante da liberação de um pool de granulócitos e aumento da liberação destas células pela medula óssea (Persson 1983). Acredita-se que a mudança na cinética leucocitária, manifestada pelo aumento da rela- 
ção neutrófilo/linfócito, está relacionada com o aumento da concentração de hormônio adrenocortical, principalmente, cortisol no sangue (Jensen-Waern et al. 1999, Robson et al. 2003). Este hormônio não estimula somente a produção de neutrófilo pela medula óssea e sua liberação para o sangue periférico, mas também inibe a migração destas células para o espaço intravascular (Pyne 1994) e interfere no número de linfócitos circulantes (Shinkai et al. 1996).

Neste estudo, os animais apresentaram aumento do número de leucócitos e neutrófilos e redução do número de linfócitos após o exercício, caracterizando o estresse fisiológico adquirido pelos mesmos durante o exercício físico. Contudo, os valores destes parâmetros permaneceram dentro dos limites fisiológicos antes e após o exercício. Segundo Thrall et al. (2007), o estresse também pode gerar eosinopenia e monocitose. Os animais estudados apresentaram redução do número de eosinófilos e aumento do número de monócitos após o exercício, entretanto, com permanência dos valores dentro dos limites de normalidade.

Para alguns pesquisadores a intensidade do exercício influencia diretamente no aumento do número de leucócitos totais e linfócitos no sangue (Rossdale et al. 1982, Snow et al. 1983b). Porém, no presente estudo não houve variação no leucograma entre os Grupos A, B, C e D. Além disso, não ocorreu influência do sexo nos índices hematológicos após o exercício, corroborando com os achados de Lacerda et al. (2006) e Krumrych (2006). Este, entretanto, relatou valores da série vermelha maiores em garanhões em comparação às éguas antes de se iniciar o treinamento, e atribuiu às maiores concentrações de testosterona e cortisol nos garanhões.

\section{CONCLUSÃO}

Com base nos resultados obtidos conclui-se que a prova de Team Penning ocasiona alterações hematológicas com interferência da frequência do exercício, independente do sexo, e com os valores da maioria dos parâmetros hematológicos permanecendo nos limites fisiológicos para a espécie.

\section{REFERÊNCIAS}

Aguilera-Tejero E., Estepa J.C., López I., Bas S., Mayer-Valor R. \& Rodríguez M. 2000. Quantitative analysis of acid-base balance in show jumpers before and after exercise. Res. Vet. Sci. 68(2):103108.

Andrews F.M., Geiser D.R., White S.L., Williamson L.H., Maykuth P.L. \& Green E.M. 1995. Haematological and biochemical changes in horses competing in a 3 Star horse trial and 3-day-event. Equine Vet. J. 20(Suppl.):57-63.

Ayres M., Ayres Júnior M., Ayres D.L. \& Santos A.A.S. 2005. Bioestat 4.0. Aplicações estatísticas nas áreas das ciências biomédicas. Sociedade Mamiaurá, Imprensa Oficial do Estado do Pará, Belém, PA.

Balarin M.R.S., Lopes R.S., Kohayagawa A., Laposy C.B. \& Fonteque J.H. 2006. Valores da Amplitude de Distribuição do Tamanho dos Eritrócitos (RDW) em eqüinos Puro Sangue Inglês (PSI) submetidos a exercícios de diferentes intensidades. Braz. J. Vet. Res. Anim. Sci. 43(5):637-641.
Balarin M.R.S., Lopes R.S., Kohayagawa A., Laposy C.B. \& Fonteque J.H. 2005. Avaliação da glicemia e da atividade sérica de aspartato aminotransferase, creatinoquinase, gama-glutamiltransferase e lactato desidrogenase em eqüinos puro sangue inglês (PSI) submetidos a exercícios de diferentes intensidades. Semin. Ciênc. Agrar. 26(2):211-218.

Boucher J.H., Ferguson E.W., Wilhelmsen C.L., Statham N. \& McMeekin R.R. 1981. Erythrocyte alterations endurance exercise in horses. J. Appl. Physiol. 51(1):131-134.

Ferraz G.C., Teixeira-Neto A.R., D'Angelis F.H.F., Lacerda Neto J.C., Queiroz Neto A. 2009. Alterações hematológicas e cardíacas em cavalos Árabes submetidos ao teste de esforço crescente em esteira rolante. Braz. J. Vet. Res. Anim. Sci. 46(6):431-437.

Ferreira Neto J.M., Viana E.S. \& Magalhães L.M. 1982. Patologia Clínica Veterinária. Rabelo, Belo Horizonte. 293p.

Gómez C., Petrón P., Andaur M., Pérez R. \& Matamoros R. 2004. Medición post-ejercicio de variables fisiológicas, hematológicas y bioquímicas en eqüinos da salto Holsteiner. Revta Cient. 14(3):244253.

Hanzawa K., Kai M., Hiraga A. \& Watanabe S. 1999. Fragility of red cells during exercise is affected by blood $\mathrm{pH}$ and temperature. Equine Vet. J. 30(Suppl.):610-611.

Harris P. \& Snow D.H. 1992. Plasma potassium and concentrations in Thoroughbred horses during exercise of varying intensity. Equine Vet. J. 24(3):220-225

Harris P. \& Snow D.H. 1988. The effects of high-intensity exercise on the plasma concentration of lactate, potassium and other electrolytes. Equine Vet. J. 20(2):109-113.

Horohov D.W., Keadle T.L., Pourciau S.S., Littlefield-Chabaud M.A., Kamerling S.G., Keowen M.L., French D.D. \& Melrose P.A. 1996. Mechanism of exercise-induced augmentation of lymphokine activated killer (LAK) cell activity in the horse. Vet. Immunol. Immunopathol. 53(3/4):221-233.

Iversen P.O., Stokland A., Rolstad B. \& Benestad H.B. 1994. Adrenalineinduced leucocytosis: Recruitment of blood cells from rat spleen, bone marrow and lymphatics. Eur. J. Appl. Physiol. Occup. Physiol. 68(3):219-227

Jain N.C. 1993. Essentials of Veterinary Hematology. Lea and Febiger, Philadelphia. 417p.

Jensen-Waern M., Lindberg A., Johannisson A., Gröndahl G., Lindgren J.A. \& Essén-Gustavsson B. 1999. The effects of an endurance ride on metabolism and neutrophil function. Equine Vet. J. 30(Suppl.):605609.

Korhonen P.A.S., Lilius E.M., Hyyppä S., Räsänen L.A. \& Pösö A.R. 2000. Production of reactive oxygen species in neutrophils after repeated bouts of exercise in standardbred trotters. J. Vet. Med. A, Physiol. Pathol. Clin. Med. 47(9):565-573.

Krumrych W. 2006. Variability of clinical and haematological indices in the course of training exercise in jumping horses. Bull. Vet. Inst. Pulawy 50:391-396.

Lacerda L., Campos R., Sperb M., Soares E., Barbosa P., Godinho E., Ferreira R., Santos V. \& González F.D. 2006. Hematologic and biochemical parameters in three high performance horse breeds southern Brazil. Arch. Vet. Sci. 11(2):40-44.

McClay C.B., Weiss D.J., Smith C.M. \& Gordon B. 1992. Evaluation of hemorheologic variables as implications for exercise-induced pulmonary hemorrhage in racing thoroughbreds. Am. J. Vet. Res. 53(8):1380-1385.

McKeever K.H., Hinchcliff K.W., Reed S.M. \& Robertson J.T. 1993. Plasma constituents during incremental treadmill exercise in intact and splenectomised horses. Equine Vet. J. 25(3):233-236.

Muñoz A., Riber C., Trigo P. \& Castejón F. 2008. Erythrocyte indices in relation to hydration and electrolytes in horses performing exercises of different intensity. Com. Clin. Pathol. 17(4):213-220. 
Orozco C.A.G. 2007. Respostas hematológicas e bioquímicas de equinos da raça Puro Sangue Árabe em testes de esforço progressivo realizados em esteira rolante durante a fase de treinamento e em prova de enduro a campo. Tese de Doutorado, Universidade Estaudal Paulista "Júlio de mesquita Filho", Jaboticabal, SP. 112p.

Orozco C.A.G., Martins C.B., D'Angelis F.H.F., Freitas E.V.V., Christovão F.G., Queiroz Neto A. \& Lacerda Neto J.C. 2006. Efeito do exercício sobre variáveis hematológicas de equinos antes e após participação em prova de enduro de $40 \mathrm{~km}$. Ars Vet. 22(3):179-183.

Pellegrini-Masini A., Baragli P., Tedeschi D., Lubas G., Martelli F., Gavazza A. \& Sighieri C. 2000. Behaviour of mean erythrocyte volume during submaximal treadmill exercise in the horse. Comp. Haematol. Int. 10(1):38-42.

Persson S.G.B. 1983. The significance of haematological data in the evaluation of soundness and fitness in the horse, p.324-327. In: Snow D.H., Persson S.G.B. \& Rose R.J. (Eds), Equine Exercise Physiology. Granta, Cambridge, UK.

Piccione G., Assenza A., Fazio F., Giudice E. \& Caola G. 2001. Different periodicities of some haematological parameters in exercise-loaded athletic horses and sedentary horses. J. Equine Sci. 12(1):17-23.

Pyne D.B. 1994. Regulation of neutrophil function during exercise. Sports Med. 17(4):245-258.

Robson P.J., Alston T.D. \& Myburgh K.H. 2003. Prolonged suppression of the innate immune system in the horse following an $80 \mathrm{~km}$ endurance race. Equine Vet. J. 35(2):133-137.

Rose R.J. 1982. Haematological changes associated with endurance exercise. Vet. Rec. 110(8):175-177.

Rose R.J., Allen J.R., Hodgson D.R., Stewart J.H. \& Chan W. 1983. Responses to submaximal treadmill exercise and training in the horse: changes in haematology, arterial blood gas and acid base measure- ments, plasma biochemical values and heart rate. Vet. Rec. 113(26/ 27):612-618.

Rossdale P.D., Burguez P.N. \& Cash R.S. 1982. Changes in blood neutrophil/lymphocyte ratio related to adrenocortical function in the horse. Equine Vet. J. 14(4):293-298.

Santos V.P. 2006. Variações hemato-bioquímicas em eqüinos de salto submetidos a diferentes protocolos de exercício físico. Dissertação de Mestrado em Ciências Veterinárias, Universidade Federal do Rio Grande do Sul, Porto Alegre. 94p.

Shinkai S., Watanabe S., Asai H. \& Shek P.N. 1996. Cortisol response to exercise and post-exercise suppression of blood lymphocyte subset counts. Int. J. Sports Med. 17(8):597-603.

Smith J.E., Erickson H.H., Debowes R.M. \& Clark M. 1989. Changes in circulating equine erythrocytes induced by brief, high-speed exercise. Equine Vet. J. 21(6):444-446.

Snow D.H., Mason D.K., Ricketts S.W. \& Douglas T.A. 1983a. Postrace blood biochemistry in Thoroughbreds, p.389-399. In: Snow D.H., Persson S.G.B. \& Rose R.J. (Eds), Equine Exercise Physiology. Granta, Cambridge, UK.

Snow D.H., Ricketts S.W. \& Mason D.K. 1983b. Haematological response to racing and training exercise in Thoroughbred horses, with particular reference to the leucocyte response. Equine Vet. J. 15(2):149-154

Thrall M.A., Baker D.C., Campbell T.W., DeNicola D., Fettman M.J., Lassen E.D., Rebar A. \& Weiser G. 2007. Interpretação da resposta leucocitária nas doenças, p.127-140. In: Ibid. (Eds), Hematologia e Bioquímica Clínica Veterinária. Roca, São Paulo.

Voss B., Mohr E. \& Krzywanek H. 2002. Effects of aqua-treadmill exercise on selected blood parameters and on heart-rate variability of horses. J. Vet. Med. A, Physiol. Pathol. Clin. Med. 49(3):137-143. 